\title{
Improving radiofrequency power and specific absorption rate management with bumped transmit elements in ultra-high field MRI
}

\author{
Alireza Sadeghi-Tarakameh ${ }^{1,2,3}$ (i) ｜ Gregor Adriany $^{3}$ ｜ Gregory J. Metzger ${ }^{3}$ | \\ Russell L. Lagore $^{3}$ ｜ Steve Jungst ${ }^{3} \quad$ | Lance DelaBarre ${ }^{3}$ | \\ Pierre-Francois Van de Moortele ${ }^{3}$ (D) | Kamil Ugurbil ${ }^{3} \quad$ Ergin Atalar ${ }^{1,2}$ (D) | Yigitcan Eryaman $^{3}$ \\ ${ }^{1}$ Department of Electrical and Electronics Engineering, Bilkent University, Ankara, Turkey \\ ${ }^{2}$ National Magnetic Resonance Research Center (UMRAM), Ankara, Turkey \\ ${ }^{3}$ Center for Magnetic Resonance Research (CMRR), University of Minnesota, Minneapolis, Minnesota, USA
}

\begin{abstract}
Correspondence
Alireza Sadeghi-Tarakameh, National Magnetic Resonance Research Center (UMRAM) \& Department of Electrical and Electronics Engineering, Bilkent University, 06800 Bilkent, Ankara, Turkey.

Email: alireza@ee.bilkent.edu.tr

Funding information

National Institutes of Health, Grant/Award Number: U01 EB025144; National Institute of Biomedical Imaging and Bioengineering, Grant/Award Number: P41 EB027061
\end{abstract}

Purpose: In this study, we investigate a strategy to reduce the local specific absorption rate (SAR) while keeping $\mathrm{B}_{1}^{+}$constant inside the region of interest (ROI) at the ultra-high field $\left(\mathrm{B}_{0} \geq 7 \mathrm{~T}\right) \mathrm{MRI}$.

Methods: Locally raising the resonance structure under the discontinuity (i.e., creating a bump) increases the distance between the accumulated charges and the tissue. As a result, it reduces the electric field and local SAR generated by these charges inside the tissue. The $\mathrm{B}_{1}^{+}$at a point that is sufficiently far from the coil, however, is not affected by this modification. In this study, three different resonant elements (i.e., loop coil, snake antenna, and fractionated dipole [FD]) are investigated. For experimental validation, a bumped FD is further investigated at 10.5T. After the validation, the transmit performances of eight-channel arrays of each element are compared through electromagnetic (EM) simulations.

Results: Introducing a bump reduced the peak 10g-averaged SAR by 21, 26, 23\% for the loop and snake antenna at 7T, and FD at 10.5T, respectively. In addition, eight-channel bumped FD array at $10.5 \mathrm{~T}$ had a $27 \%$ lower peak $10 \mathrm{~g}$-averaged SAR in a realistic human body simulation (i.e., prostate imaging) compared to an eightchannel FD array.

Conclusion: In this study, we investigated a simple design strategy based on adding bumps to a resonant element to reduce the local SAR while maintaining $\mathrm{B}_{1}^{+}$inside an ROI. As an example, we modified an FD and performed EM simulations and phantom experiments with a $10.5 \mathrm{~T}$ scanner. Results show that the peak $10 \mathrm{~g}$-averaged SAR can be reduced more than $25 \%$.

K E Y W O R D S

10.5 Tesla, bumped transmitter, MRI, radiofrequency safety, ultra-high field 


\section{1 | INTRODUCTION}

Demand for the ultra-high field (UHF, $\mathrm{B}_{0} \geq 7 \mathrm{~T}$ ) MRI is continuously increasing due to its numerous benefits such as a significant increase in signal-to-noise ratio ${ }^{1-6}$ (SNR) and improved susceptibility contrast. ${ }^{7,8}$ However, with an increasing static magnetic field $\left(\mathrm{B}_{0}\right)$, electromagnetic (EM) wavelengths decrease resulting in destructive and constructive interferences of radiofrequency (RF) magnetic fields causing degradation in overall image quality. In addition, constructive interference of the RF electric field can lead to elevated local specific absorption rate (SAR) hot spots, which are an important patient safety concern at UHF. Nevertheless, it is shown that multichannel transmit arrays (TxArrays) with improved RF performance can be used to alleviate both issues when optimal excitation strategies are used. $^{9,10}$

In the literature, various types of the transmitting elements, such as loop coils, ${ }^{11-16}$ transmission lines, ${ }^{6,9,17-19}$ and dipole-like structures, ${ }^{20-26}$ are investigated for UHFMRI. Thalhammer et $\mathrm{al}^{11}$ used TxArray of loop coils for cardiac imaging at 7T. Adriany et $\mathrm{al}^{9}$ used an array of the transmission lines for head imaging at $7 \mathrm{~T}$. Vaughan et $\mathrm{al}^{18}$ built a transverse electromagnetic (TEM) coil for whole-body imaging at $7 \mathrm{~T}$. Raaijmaker et $\mathrm{al}^{20,21}$ introduced single-side adapted and fractionated dipole as new transmitter elements for body imaging at 7T. Steensma et $\mathrm{al}^{22,23}$ improved the SAR performance of the dipoles at $7 \mathrm{~T}$ and $10.5 \mathrm{~T}$ by introducing the snake antenna. Vaughan et $\mathrm{al}^{6}$ used a TEM coil for the head imaging at 9.4T. Shajan et $\mathrm{al}^{13}$ performed head imaging at $9.4 \mathrm{~T}$ using the array of loop elements. Erturk et $\mathrm{al}^{24}$ proposed to use an array of fractionated dipoles for torso imaging at $10.5 \mathrm{~T}$. RF coil development is essential for realizing the full potential of UHF-MRI. Improving the performance of RF coil arrays improves UHF imaging as demonstrated in numerous studies in the literature.

In this study, we propose to modify the geometry of individual coil elements by placing a bump underneath the discontinuities (i.e., all lumped elements and excitation ports) on the coil. ${ }^{27}$ This reduces the peak local SAR while maintaining comparable $\mathrm{B}_{1}^{+}$within the target region of interest (ROI). As proof of concept, EM simulations were performed for three different types of coils: the loop coil and snake antenna at $7 \mathrm{~T}$, and the fractionated dipole at 10.5T. In addition, a bumped fractionated dipole is constructed and experimentally compared to a fractionated dipole design previously proposed for $10.5 \mathrm{~T}^{24}$ Moreover, two eight-channel body arrays are simulated using the bumped and regular fractionated dipoles and their Tx performance are compared to each other.

\section{2 | METHODS}

According to Equation 1, the continuity equation in the electromagnetic theory, spatial variation of the current density $\bar{J}$ produces electrical charges.

$$
\nabla \cdot \bar{J}=-j \omega \rho
$$

where $\rho$ denotes the electrical charge density. Based on this basic principle we can predict that a high SAR region would occur underneath a conductor discontinuity on an RF transmitter. In other words, the discontinuity on the pathway of the current leads to charge accumulation, which results in an elevated electric field (so-called conservative electric field) and high local SAR in the tissue. As a solution for this RF safety problem, we propose to place a bump underneath the discontinuities and increase the distance between the accumulated charges and the body. This simple modification reduces the conservative electric field inside the tissue. On the other hand, the $\mathrm{B}_{1}^{+}$at a point which is sufficiently far from the coil is not affected by this modification.

\section{1 | Numerical optimization of the bump geometry}

As a proof concept, simple bumps were placed underneath the discontinuities of a loop coil, ${ }^{11}$ the snake antenna, ${ }^{22}$ and the fractionated dipole ${ }^{24}$ as shown in Figure 1A-F. To determine the optimum height of the bump in each case, a deep-body target (e.g., prostate imaging) is defined, and accordingly, the following optimization problem is investigated by sweeping the height in a reasonable range.

$$
\begin{aligned}
& \text { minimize }[\text { peak } 10 \mathrm{~g} \text {-averaged } \mathrm{SAR}] \\
& \text { subject to }\left[\mathrm{B}_{1}^{+}\right. \text {remaining constant at the target range } \\
& \text { (i.e., } 50 \text { to } 120 \mathrm{~mm} \text { depth), compared to the } \\
& \text { original structure] }
\end{aligned}
$$

The height of the bump was swept from $5 \mathrm{~mm}$ to $35 \mathrm{~mm}$ for the loop coil (7T), $60 \mathrm{~mm}$ for the snake antenna (7T), and $60 \mathrm{~mm}$ for the fractionated dipole (10.5T), moreover according to the original structures, ${ }^{11,22,24}$ the element-to-phantom separations of $15 \mathrm{~mm}, 20 \mathrm{~mm}$, and $20 \mathrm{~mm}$ were determined respectively. The EM simulations were performed using a numerical EM solver HFSS 18.1 (ANSYS, Canonsburg, PA, USA) based on the finite-element method (FEM). In all cases, conductors were placed on an FR4 printed circuit board (PCB) which was mounted on a block of polyethylene terephthalate glycol (PETG). A uniform rectangular phantom $\left(200 \times 200 \times 400 \mathrm{~mm}^{3}\right)$ with electrical properties 

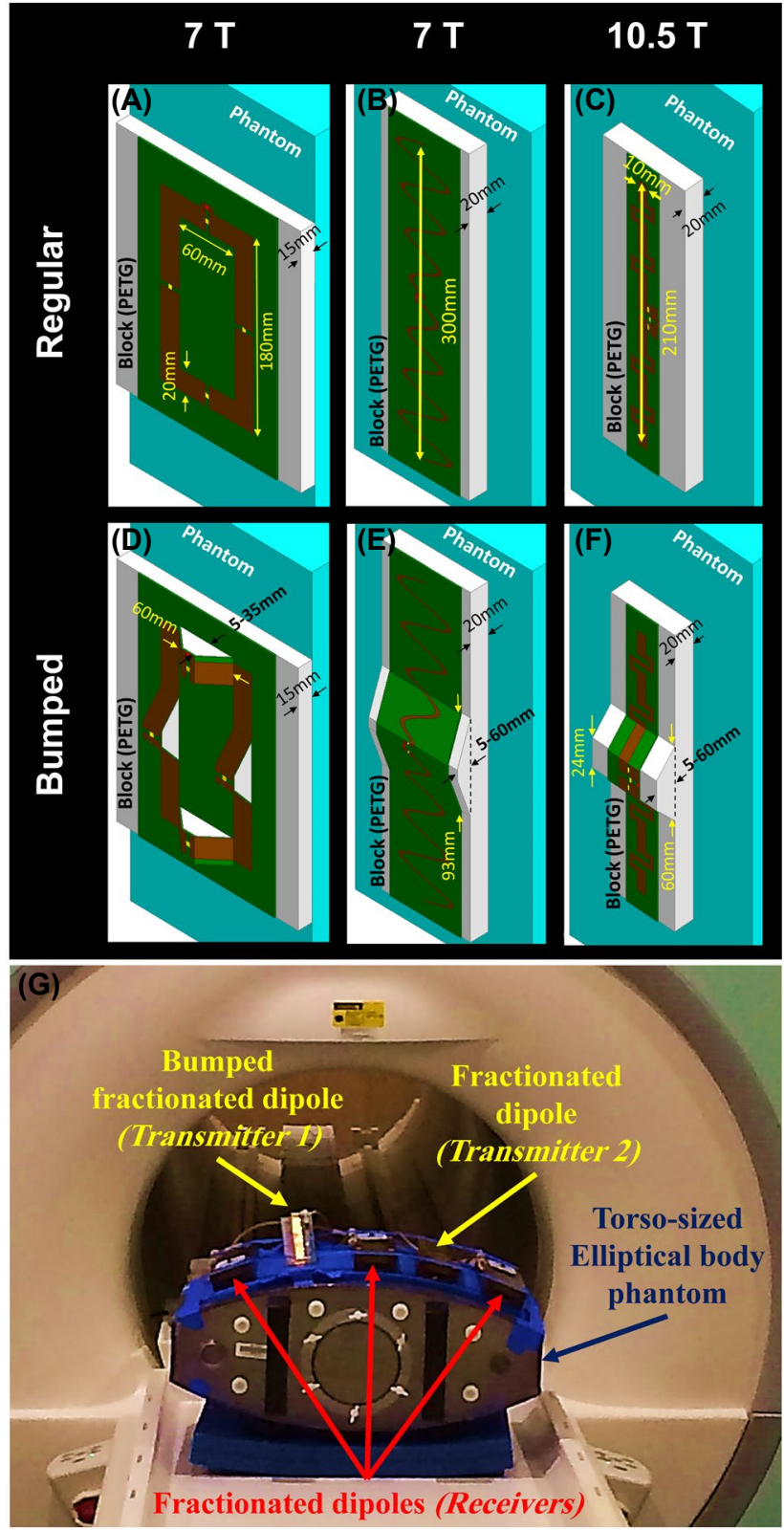

F I G U RE 1 Regular vs. bumped elements. A-C, Loop coil at 7T, snake antenna at 7T, and fractionated dipole at 10.5T. D-F, Bumped loop coil, snake antenna, and fractionated dipole. G, The experimental setup used to compare the transmit performance of the bumped and regular fractionated dipole. The experiment was conducted on a $10.5 \mathrm{~T}$ scanner. The experimental set-up is also modeled in the simulation environment to perform a comparison with the experimental data

similar to the average values of the human body $\left(\varepsilon_{\mathrm{r}}=36\right.$, $\sigma=0.42 \mathrm{~S} / \mathrm{m}$ ) were used in the simulations for optimization.

\subsection{Experimental element-wise comparison}

Both the bumped and regular FDs were constructed and matched to obtain a reflection coefficient $-15 \mathrm{~dB}$ or less. Both dipoles consisted of conductors on an FR4 PCB mounted on a PETG block with the thickness of $10 \mathrm{~mm}$. The regular ${ }^{24}$ and the bumped fractionated dipoles, the latter with an optimized bump height using the methods detailed above, were positioned on a torso-sized elliptical body phantom $(450 \times 180 \times$ $290 \mathrm{~mm}^{3}$ ) (Figure 1G) ${ }^{28}$ The phantom was filled with a $\mathrm{NaCl}$ doped hydroxyethyl cellulose (HEC) gel with electrical properties of $\varepsilon_{\mathrm{r}}=78.3$ and $\sigma=0.66 \mathrm{~S} / \mathrm{m}$. The two FD elements being compared were used as transmitters in this setup while an additional three FD elements were placed on the phantom as receivers only (Figure 1G).

Experimental studies with the above setup were conducted on a whole-body 10.5T Magnet (Agilent Technologies, Oxford, UK) and associated imaging system (Siemens Healthineers, Erlangen, Germany). The scanner is equipped with a 16-channel parallel transmit system with each channel driven by a 2-kW RF power amplifiers (Stolberg HF-Technik AG, Stolberg, Germany). Transmit $B_{1}^{+}$maps were acquired using the actual flip-angle imaging (AFI) technique. ${ }^{29}$ Temperature mapping was performed using magnetic resonance thermometry ${ }^{30}$ (MRT) based on the proton resonance offset method ${ }^{31}$ with a 3D multi-echo gradient-echo sequence. SAR values were calculated by finding the slope of the initial part of the heating curve and multiplying with the heat capacity of the HEC gel $\left(4386 \mathrm{~J} / \mathrm{kg} /{ }^{\circ} \mathrm{K}\right)$. The experimental $\mathrm{B}_{1}^{+}$and SAR maps were compared against EM simulations of the same configuration performed using the HFSS.

\section{3 | Eight-channel body TxArray coils: numerical simulations}

To demonstrate the potential of the bumped geometry in a multichannel excitation scenario, simulations were performed with arrays of the two element designs. Two separate eight-channel TxArrays comprised of bumped or regular elements were simulated using a uniform phantom and a realistic human body model. In both cases four anterior and four posterior dipoles were positioned circumferentially around the load. The blocks used in these simulations were $10 \mathrm{~mm}$ thick instead of $20 \mathrm{~mm}$ (i.e., used through the optimization procedure) in order to alleviate the lack of physical space due to the bore size limitations.

In the phantom simulations, all elements were matched to obtain a reflection coefficient of $-20 \mathrm{~dB}$ or less. We maximized the $\mathrm{B}_{1}^{+}$-power efficiency at a prostate sized region at the center of the phantom using a phase-only shimming method, without consideration of the local SAR. ${ }^{32}$ The total input power was adjusted to enforce the same average $\mathrm{B}_{1}^{+}$ value inside the ROI for both TxArrays.

For the body model simulations, the torso of the realistic human body model Duke of the virtual population was used (voxel size, $2 \times 2 \times 2 \mathrm{~mm}^{3}$; height, $1.77 \mathrm{~m}$; weight, $70.3 \mathrm{~kg}$ ), as shown in Figure 2C,D. In this case, EM simulations were 


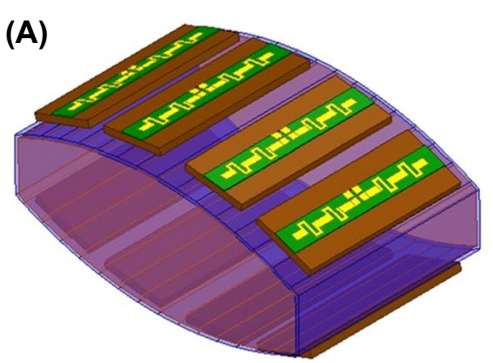

(B)
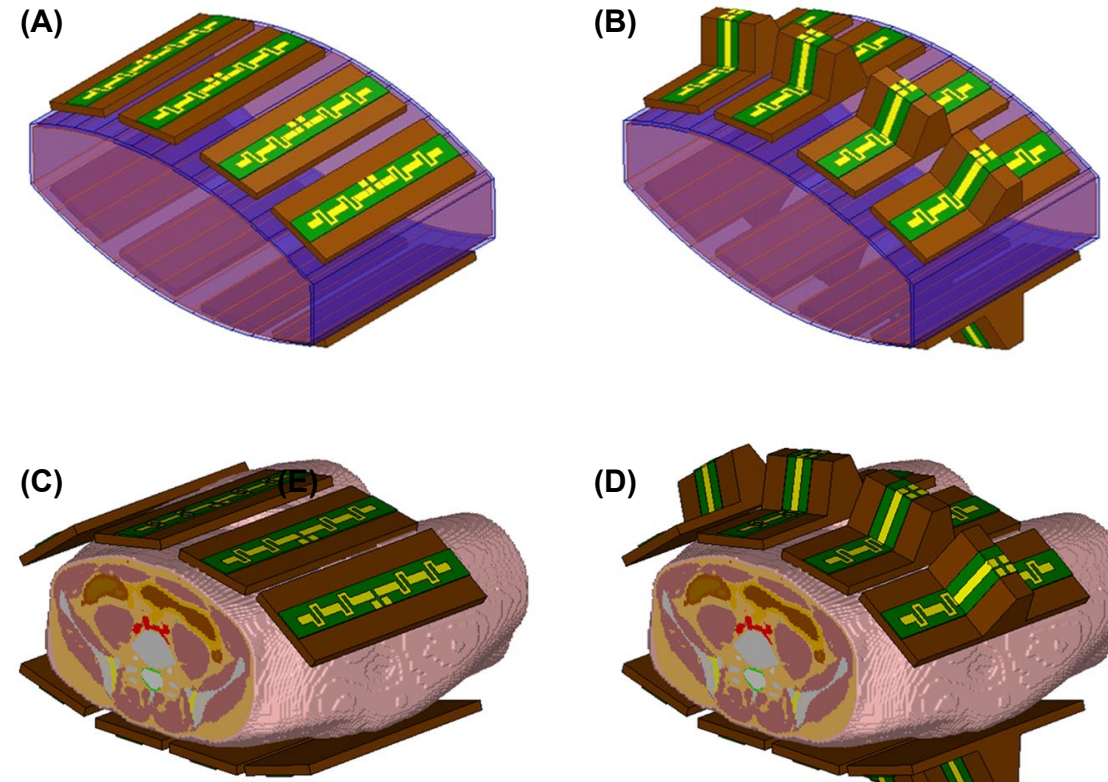

(A)
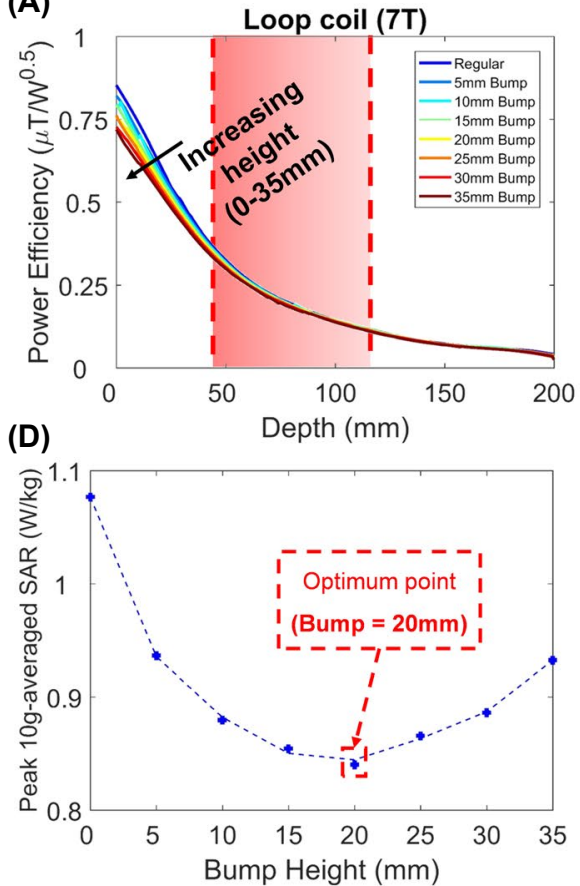

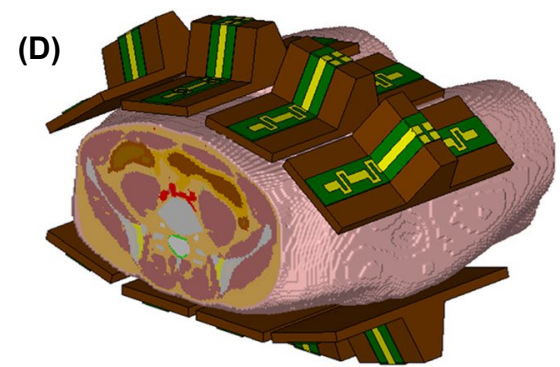

(B)

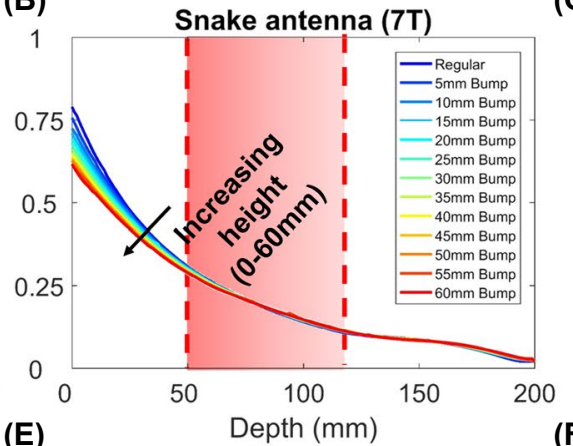

(C)

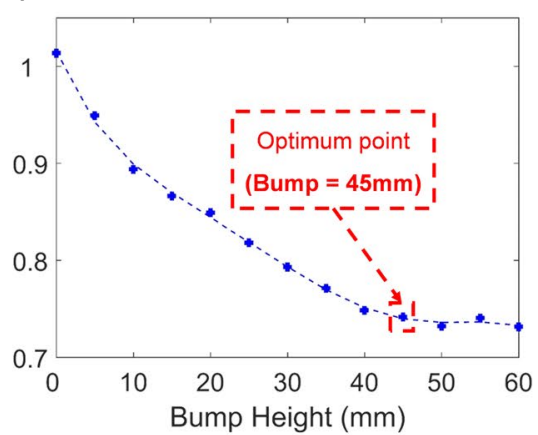

FIGURE 2 Comparison between the eight-channel TxArray of regular and bumped fractionated dipoles. An eightchannel TxArray coil of regular (A) and bumped (B) fractionated dipoles in the presence of a uniform elliptic phantom. An eight-channel TxArray coil of regular (C) and bumped (D) fractionated dipoles in the presence of a realistic human body model

F I G U R E 3 Optimizing bump height. A-C, $\mathrm{B}_{1}^{+}$-power efficiencies of a loop coil, a snake antenna, and a fractionated dipole are calculated for various bump heights and sampled on a line perpendicular to the surface of the coil passing through the center of the coil. D-F, Peak $10 \mathrm{~g}$-averaged SAR for each bump geometry is also calculated while the input power is adjusted to achieve the reference $\mathrm{B}_{1}^{+}$at the depth of $80 \mathrm{~mm}$

performed in CST studio 2018 (CST, Darmstadt, Germany) using a time-domain solver based on the finite-integration technique (FIT). All coil elements were matched to obtain a reflection coefficient of $-15 \mathrm{~dB}$ or less. A phase-only shimming optimization was applied to maximize the $\mathrm{B}_{1}^{+}$-power efficiency on the prostate without consideration of the local SAR. Similar to the phantom simulations, the total input power was adjusted to enforce the same average $\mathrm{B}_{1}^{+}$value inside the ROI for both TxArrays.

\section{$3 \mid$ RESULTS}

\subsection{Numerical optimization of the bump geometry}

Figure $3 \mathrm{~A}-\mathrm{C}$ show the $\mathrm{B}_{1}^{+}$-power efficiency results (i.e., $B_{1}^{+} / \sqrt{\text { input power }}$ sampled on a line perpendicular to the coil surface and passing through the coil's center for the loop coil, the snake antenna, and the fractionated dipole, respectively. The target range (i.e., 50 to $120 \mathrm{~mm}$ depth in the 
phantom) is shown with a shaded region in the figure. As can be seen, increasing the height of the bumps results in reduced $\mathrm{B}_{1}^{+}$-power efficiency at a region close to the coil. On the other hand, bumps did not have a significant effect on the $\mathrm{B}_{1}^{+}$-power efficiency at the target region. In Figure 3D-F, the $\mathrm{B}_{1}^{+}$at a depth of $80 \mathrm{~mm}$ (i.e., the average distance from the transmit element to the prostate) corresponding to the regular structures was chosen as a reference. Then, the input power in the case of each height was adjusted such that the reference $\mathrm{B}_{1}^{+}$value has been achieved. Finally, the peak $10 \mathrm{~g}$-averaged SARs were determined and plotted for the adjusted input powers. As indicated on the graphs, the height of the bump that minimizes peak $10 \mathrm{~g}$-averaged SAR can be chosen as the optimum height. For the loop coil, there is a well-defined global optimum height. However, for the snake antenna and the fractionated dipole, the peak SAR plot reaches a plateau at a certain bump height. This allows us to pick a smaller bump size for those designs, improving the ease of fabrication and patient comfort without sacrificing SAR efficiency.

\subsection{Experimental element-wise comparison}

Using the phantom configuration of Figure $1 \mathrm{G}$ and the optimized bump height of 50 for the FD determined during optimization, simulation and experimental $\mathrm{B}_{1}^{+}$-power efficiencies were determined experimentally and from simulation as shown in Figure 4A,B, respectively. The $\mathrm{B}_{1}^{+}$-power efficiencies were sampled along the dashed lines perpendicular to the surface of the phantom and passing through the center of the dipoles in Figure 4A,B, then they were plotted with respect to the depth inside the phantom as shown in Figure 4C. Both simulation and experimental results demonstrate that the $\mathrm{B}_{1}^{+}$ -power efficiency of the bumped FD approaches the regular FD and becomes almost identical at a depth $\sim 60 \mathrm{~mm}$.

The simulated and measured 10g-averaged SAR maps determined from MRT are given in Figure 4D,E, respectively.

Figure $4 \mathrm{~F}$ shows the simulation results for the $\mathrm{B}_{1}^{+}$-SAR efficiency $\left(B_{1}^{+} / \sqrt{\text { peak } S A R_{10 g}}\right)$ of the bumped FD relative to regular FD plotted with respect to depth. The bumped FD achieved a $25 \%$ improvement in $\mathrm{B}_{1}^{+}$-SAR efficiency at all depths greater than $50 \mathrm{~mm}$.

\section{3 | Eight-channel body TxArray coils: numerical simulations}

The simulated $\mathrm{B}_{1}^{+}$-maps inside a uniform elliptic phantom obtained using the eight-channel TxArray of fractionated and bumped dipoles are shown in Figure 5A,B, respectively. The maps correspond to the phase-only shimming solution for the prostate-size ROI indicated by the black square. The input powers for both arrays were adjusted to achieve the same average $\mathrm{B}_{1}^{+}(1 \mu \mathrm{T})$ inside the ROI.
(A)

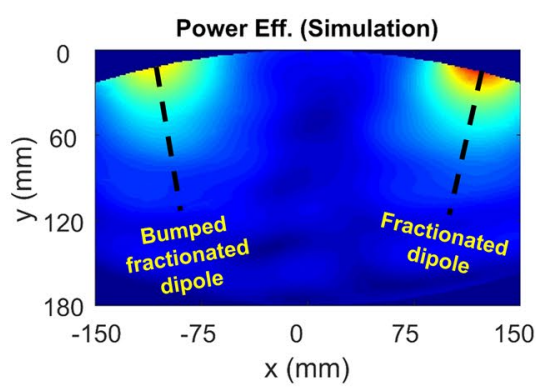

(D)

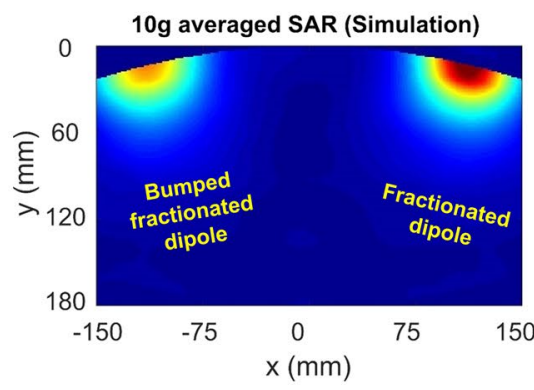

(B)

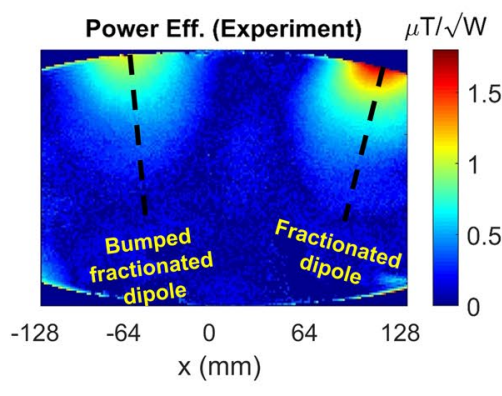

(E)

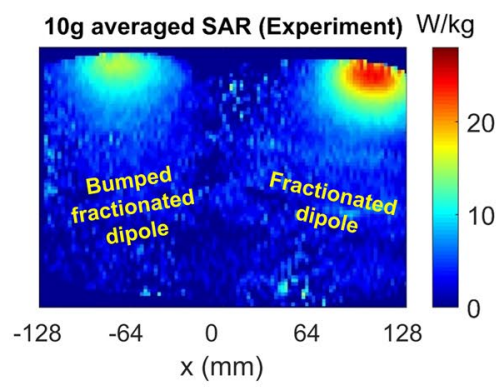

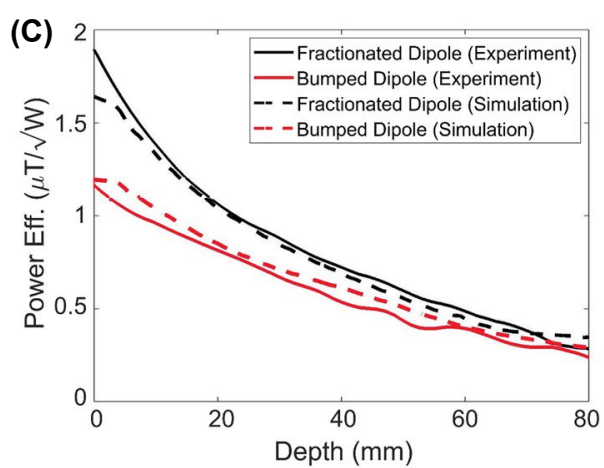

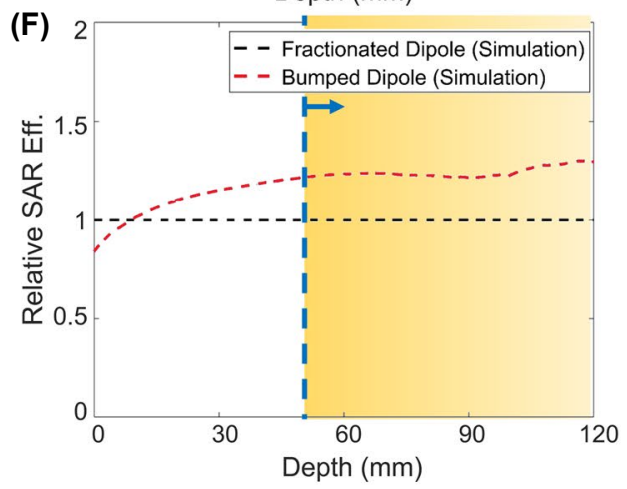

F I G U RE 4 Simulation and experimental results for individual fractionated and bumped dipoles. A-B, The $\mathrm{B}_{1}^{+}$-power efficiencies inside a uniform phantom. $C$, The simulated and experimentally measured $\mathrm{B}_{1}^{+}$-power efficiencies on the dashed lines shown in Figure 4A,B. D-E, The $10 \mathrm{~g}$-averaged SAR. F, The $\mathrm{B}_{1}^{+}$-SAR efficiency of the bumped element (calculated relative to the regular fractionated dipole) on the dashed lines shown in Figure 4A 

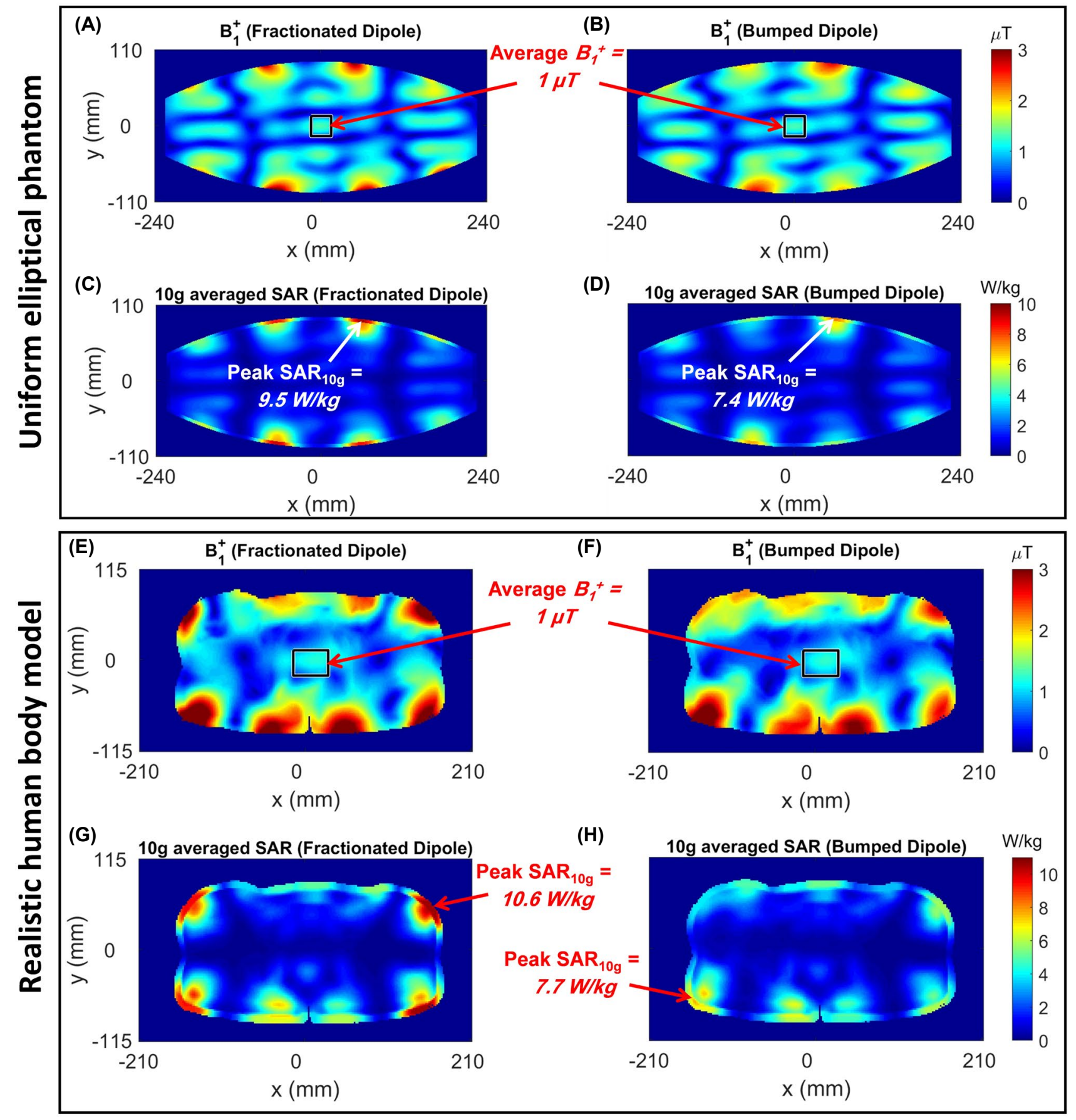

F I G U RE 5 EM simulations corresponding to eight-channel fractionated and bumped dipoles in the presence of a uniform elliptical phantom and realistic human body model (Duke). Phase-only shimming solutions for a prostate-size ROI at the center of the uniform elliptical phantom (A,B) and corresponding 10g-averaged SAR maps on the transverse plane, which includes the peak local SAR (C,D). Phase-only shimming solutions for the prostate (E,F) and corresponding 10g-averaged SAR maps on the transverse plane, which includes the peak local SAR (G,H)

The $10 \mathrm{~g}$-averaged SAR maps corresponding to the identical excitations used to obtain the $\mathrm{B}_{1}^{+}$-maps in Figure 5A,B are shown in Figure 5C,D. The presented SAR map is over the transverse plane which includes the location of peak local SAR. The bumped dipole array had a 22\% lower peak SAR than the regular dipole array, yet achieved the same $\mathrm{B}_{1}^{+}$on the prostate-size ROI.

Similarly, the simulated $\mathrm{B}_{1}^{+}$-maps inside the realistic human body model (Duke) are shown in Figure 5E,F. The phase-only shimming optimization was performed in the prostate on the transverse slice passing through the center of the dipoles.

The corresponding 10g-averaged SAR map on a transverse plane including the location of peak local SAR. Based on these results, the bumped dipole array can achieve a $27 \%$ reduction in peak $10 \mathrm{~g}$-averaged SAR while maintaining the same $\mathrm{B}_{1}^{+}$-power efficiency in the prostate. 


\section{4 | DISCUSSION AND CONCLUSIONS}

In this study, we investigated a simple strategy based on adding bumps to a resonant transmit element underneath the discontinuities (i.e., lumped elements and excitation port) in order to reduce the local SAR while keeping $\mathrm{B}_{1}^{+}$-power efficiency constant inside an ROI. This approach works especially well when the target of interest is relatively far away from the coil (i.e., prostate imaging). A similar advantage may also be present for head imaging studies at UHF, ${ }^{33,34}$ especially for spectroscopy studies that aim to maximize $\mathrm{B}_{1}^{+}$-power efficiency at a single voxel near the middle of the brain. ${ }^{35}$

We demonstrated the advantage of adding bumps for SAR reduction using three transmit elements; loop coils and snake antennas at 7T, and fractionated dipoles at 10.5T. The reason for such selections was to present the advantage of adding bump in a fair and unbiased manner since the selected structures are optimized and presented in previously published works. ${ }^{11,22,24}$ This advantage is investigated for a fractionated dipole at $7 \mathrm{~T}^{21}$ as well, and the results are presented in Supporting Information Figure S1, which is available online. In principle, the approach is expected to work for all transmit elements with lumped matching and tuning elements. Optimizing arrays for the transmit performance in a collective manner is a challenging task, mainly because it requires modeling the complex interactions between individual transmit elements. As an alternative strategy, individual coil elements can be optimized for a given metric assuming that an array comprised of such elements will also yield optimal results. ${ }^{21}$ Although this conclusion is not generally true for arbitrary coil arrays, it has been shown that optimizing the individual transmitters may result in improved array performance, especially when the EM interactions between individual elements are weak. In this work, we optimized the bump height for the individual elements. This optimization resulted in an overall improvement of the array performance as demonstrated with our simulation results.

Although the $\mathrm{B}_{1}^{+}$-SAR efficiency of transmit elements was improved in the ROI by adding the bump, the power efficiency was slightly declined which indicate a degradation in SNR. The reduction in power efficiency mostly can be tackled by increasing the transmit power; however, the SNR issue may remain unsolved if the bumped elements will be used as a receive array as well. A potential solution for such a problem during reception can be using other types of receive elements along with the bumped elements (e.g., loop-dipole combination ${ }^{25}$ ).

As shown in Figure 3B, introducing the bump resulted in an increase in $\mathrm{B}_{1}^{+}$-power efficiency in the target depth-of-interest (shown with the shaded area). The snake antenna was originally designed to optimize the $\mathrm{B}_{1}^{+}$-SAR efficiency $\left(B_{1}^{+} / \sqrt{\text { peak } S A R_{10 g}}\right)$; however, $\mathrm{B}_{1}^{+}$-power efficiency was not optimized. Adding a bump to the antenna have altered the physical and effective length of the antenna resulting in an increased $\mathrm{B}_{1}^{+}$-power efficiency.

According to Figure 3, the optimal bump heights for the snake antenna and the fractionated dipole are $45 \mathrm{~mm}$ and 40 $\mathrm{mm}$, respectively, which makes the elements rather bulky. Using these elements to form a transmit array similar to Figure 2 may have some drawbacks. For example, the bore size may be a limiting physical factor especially for subjects with a higher body mass index (BMI). In addition, the conductors at the bump will be closer to the gradient shield and this may affect matching of the elements. However, both issues can be addressed by modestly sacrificing $\mathrm{B}_{1}^{+}$ -SAR efficiency and choosing a lower bump height (e.g., $30 \mathrm{~mm}$ instead of $45 \mathrm{~mm}$ for the snake antenna and $40 \mathrm{~mm}$ for the FD which reduce $\mathrm{B}_{1}^{+}$-SAR efficiency by $3.7 \%$ and $1.5 \%$, respectively).

Experimentally, we investigated the bumped fractionated dipole at $10.5 \mathrm{~T}$ comprehensively. We built regular and bumped fractionated dipoles and compared their transmit performances to validate our simulation results.

In this work, we investigated the effect of adding bumps to a resonant transmit element underneath the discontinuities (i.e., lumped elements and excitation port). The suggested modification reduced the $10 \mathrm{~g}$ - averaged local SAR $(\sim 25 \%)$ while preserving the $\mathrm{B}_{1}^{+}$-power efficiency at a distant point of interests. This preliminary work demonstrates that bumped elements are promising candidates as Tx coils for deep-targets (e.g., prostate) imaging at UHF. Future studies will focus on using the proposed topology for in vivo imaging at UHF.

\section{ACKNOWLEDGMENT}

This study was supported by the following grants: NIBIB P41 EB027061, U01 EB025144.

\section{ORCID}

Alireza Sadeghi-Tarakameh (D) https://orcid. org/0000-0001-5718-6553

Pierre-Francois Van de Moortele (iD https://orcid. org/0000-0002-6941-5947

Ergin Atalar (D) https://orcid.org/0000-0002-6874-6103

\section{REFERENCES}

1. Edelstein WA, Glover GH, Hardy CJ, Redington RW. The intrinsic signal-to-noise ratio in NMR imaging. Magn Reson Med. 1986;3:604-618.

2. Ocali O, Atalar E. Ultimate intrinsic signal-to-noise ratio in MRI. Magn Reson Med. 1998;39:462-473.

3. Cho Z-H, Kang C-K, Han J-Y, et al. Observation of the lenticulostriate arteries in the human brain in vivo using 7.0 T MR angiography. Stroke. 2008;39:1604-1606. 
4. Duyn JH, van Gelderen P, Li T-Q, de Zwart JA, Koretsky AP, Fukunaga M. High-field MRI of brain cortical substructure based on signal phase. Proc Natl Acad Sci. 2007;104:11796-11801.

5. Nakada T, Matsuzawa H, Igarashi H, Fujii Y, Kwee IL. In vivo visualization of senile-plaque-like pathology in Alzheimer's disease patients by MR microscopy on a 7T system. J Neuroimaging. 2008;18:125-129.

6. Vaughan T, DelaBarre L, Snyder C, et al. 9.4 T human MRI: Preliminary results. Magn Reson Med. 2006;56:1274-1282.

7. Abduljalil AM, Schmalbrock P, Novak V, Chakeres DW. Enhanced gray and white matter contrast of phase susceptibility-weighted images in ultra-high-field magnetic resonance imaging. J Magn Reson Imaging. 2003;18:284-290.

8. van der Zwaag W, Schäfer A, Marques JP, Turner R, Trampel R. Recent applications of UHF-MRI in the study of human brain function and structure: A review. NMR Biomed. 2016;29: 1274-1288.

9. Adriany G, Van de Moortele P-F, Wiesinger F, et al. Transmit and receive transmission line arrays for 7 Tesla parallel imaging. Magn Reson Med. 2005;53:434-445.

10. Metzger GJ, Snyder C, Akgun C, Vaughan T, Ugurbil K, Van de Moortele PF. Local B1+ shimming for prostate imaging with transceiver arrays at 7T based on subject-dependent transmit phase measurements. Magn Reson Med. 2008;59:396-409.

11. Thalhammer C, Renz W, Winter L, et al. Two-dimensional sixteen channel transmit/receive coil array for cardiac MRI at 7.0 T: Design, evaluation, and application. J Magn Reson Imaging. 2012;36:847-857.

12. Zhao W, Cohen-Adad J, Polimeni JR, et al. Nineteen-channel receive array and four-channel transmit array coil for cervical spinal cord imaging at 7T. Magn Reson Med. 2014;72:291-300.

13. Shajan G, Kozlov M, Hoffmann J, Turner R, Scheffler K, Pohmann R. A 16-channel dual-row transmit array in combination with a 31-element receive array for human brain imaging at 9.4 T. Magn Reson Med. 2014;71:870-879.

14. Wu B, Wang C, Krug R, et al. $7 \mathrm{~T}$ human spine imaging arrays with adjustable inductive decoupling. IEEE Trans Biomed Eng. 2009;57:397-403.

15. Kraff O, Bitz AK, Kruszona S, et al. An eight-channel phased array $\mathrm{RF}$ coil for spine MR imaging at $7 \mathrm{~T}$. Invest Radiol. 2009;44:734-740.

16. Avdievich NI, Giapitzakis IA, Pfrommer A, Borbath T, Henning A. Combination of surface and "vertical" loop elements improves receive performance of a human head transceiver array. Biomedicine. 2018;31:e3878.

17. Adriany G, Auerbach EJ, Snyder CJ, et al. A 32-channel lattice transmission line array for parallel transmit and receive MRI at 7 tesla. Magn Reson Med. 2010;63:1478-1485.

18. Vaughan JT, Snyder CJ, DelaBarre LJ, et al. Whole-body imaging at 7T: preliminary results. Magn Reson Med. 2009;61:244-248.

19. Snyder CJ, DelaBarre L, Metzger GJ, et al. Initial results of cardiac imaging at 7 Tesla. Magn Reson Med. 2009;61:517-524.

20. Raaijmakers AJE, Ipek O, Klomp DWJ, et al. Design of a radiative surface coil array element at $7 \mathrm{~T}$ : The single-side adapted dipole antenna. Magn Reson Med. 2011;66:1488-1497.

21. Raaijmakers AJE, Italiaander M, Voogt IJ, et al. The fractionated dipole antenna: A new antenna for body imaging at $7 \mathrm{~T}$ esla. Magn Reson Med. 2016;75:1366-1374.

22. Steensma B, Andrade AV, Klomp DW, Van den Berg CA, Luijten PR, Raaijmakers AJ. Body imaging at 7 Tesla with much lower SAR levels: an introduction of the Snake Antenna array. In: Proceedings of the 24th Annual Meeting of ISMRM, Singapore, 2016. p 0395.

23. Steensma B, van de Moortele PF, Ertürk A, et al. Introduction of the snake antenna array: Geometry optimization of a sinusoidal dipole antenna for $10.5 \mathrm{~T}$ body imaging with lower peak SAR. Magn Reson Med. 2020;84:2885-2896.

24. Ertürk MA, Wu X, Eryaman Y, et al. Toward imaging the body at 10.5 tesla. Magn Reson Med. 2017;77:434-443.

25. Ertürk MA, Raaijmakers AJE, Adriany G, Uğurbil K, Metzger GJ. A 16-channel combined loop-dipole transceiver array for 7 Tesla body MRI. Magn Reson Med. 2017;77:884-894.

26. Sadeghi-Tarakameh A, Jungst $S$, Wu X, et al. A new coil element for highly-dense transmit arrays: An introduction to non-uniform dielectric substrate (NODES) antenna. In: Proceedings of the 27th Annual Meeting of ISMRM, Montreal, QC, Canada, 2019. p 0732.

27. Sadeghi-Tarakameh A, Torrado-Carvajal A, Ariyurek C, et al. Optimizing the topography of transmit coils for SAR management. In: Proceedings of the 26th Joint Annual Meeting of ISMRMESMRMB, Paris, France, 2018. p 0297.

28. Ertürk MA, Tian J, Van de Moortele PF, Adriany G, Metzger GJ. Development and evaluation of a multichannel endorectal RF coil for prostate MRI at 7T in combination with an external surface array. J Magn Reson Imaging. 2016;43:1279-1287.

29. Yarnykh VL. Actual flip-angle imaging in the pulsed steady state: A method for rapid three-dimensional mapping of the transmitted radiofrequency field. Magn Reson Med. 2007;57:192-200.

30. Kuroda K, Oshio K, Chung AH, Hynynen K, Jolesz FA. Temperature mapping using the water proton chemical shift: A chemical shift selective phase mapping method. Magn Reson Med. 1997;38:845-851.

31. Poorter JD, Wagter CD, Deene YD, Thomsen C, Ståhlberg F, Achten E. Noninvasive MRI thermometry with the proton resonance frequency (PRF) method: in vivo results in human muscle. Magn Reson Med. 1995;33:74-81.

32. Metzger GJ, Snyder C, Akgun C, Vaughan T, Ugurbil K, de Moortele V. Local B1+ shimming for prostate imaging with transceiver arrays at $7 \mathrm{~T}$ based on subject-dependent transmit phase measurements. Magn Reson Med. 2008;59:396-409.

33. Sadeghi-Tarakameh A, DelaBarre L, Lagore RL, et al. In vivo human head MRI at $10.5 \mathrm{~T}$ : A radiofrequency safety study and preliminary imaging results. Magn Reson Med. 2019;84:484-496.

34. Sadeghi-Tarakameh A, Torrado-Carvajal A, Lagore RL, et al. Toward human head imaging at $10.5 \mathrm{~T}$ using an eight-channel transmit/receive array of bumped fractionated dipoles. In: Proceedings of the 27th Annual Meeting of ISMRM, Montreal, QC, Canada, 2019. p 0430.

35. Marjanska M, Auerbach EJ, Valabregue R, Van de Moortele PF, Adriany G, Garwood M. Localized 1H NMR spectroscopy in different regions of human brain in vivo at $7 \mathrm{~T}$ : T2 relaxation times and concentrations of cerebral metabolites. NMR Biomed. 2012;25:332-339.

\section{SUPPORTING INFORMATION}

Additional supporting information may be found online in the Supporting Information section.

FIGURE S1 Regular vs. bumped fractionated dipole at $7 \mathrm{~T}$. (A) Structure of the regular fractionated dipole used for the EM simulations. (B) Structure of the bumped fractionated dipole where the bump height was swept between $5 \mathrm{~mm}$ and 
$60 \mathrm{~mm}$. (C) $\mathrm{B}_{1}^{+}$-power efficiencies of the fractionated dipoles are calculated for various bump heights and sampled on a line perpendicular to the surface of the dipole passing through the center of the dipole. (D) Peak 10g-averaged SAR for each bump height is also calculated while the input power is adjusted to achieve the reference $\mathrm{B}_{1}^{+}$at the depth of $80 \mathrm{~mm}$. As a result, $31 \%$ reduction in the peak $10 \mathrm{~g}$-averaged SAR is achieved for a $55 \mathrm{~mm}$-bump compared to the regular structure
How to cite this article: Sadeghi-Tarakameh A, Adriany G, Metzger GJ, et al. Improving radiofrequency power and specific absorption rate management with bumped transmit elements in ultra-high field MRI. Magn Reson Med. 2020;84: 3485-3493. https://doi.org/10.1002/mrm.28382 